\title{
The recycling of wool clothes: an artificial neural network colour classification tool
}

\author{
Rocco Furferi, Lapo Governi
}

\begin{abstract}
Recycling of clothes is a straightforward approach for the supply of a coloured raw material which does not involve the cost of the colouring process. A real time and completely automated colour classification tool for woollen clothes to be recycled is proposed. The tool uses the combination of a statistical method, called matrix approach, of a self-organizing feature map (SOFM) and a feedforward backpropagation artificial neural network (FFBP ANN)-based approach, to correctly classify the clothes by respecting the selection criteria provided by human know-how.

The developed tool, which uses an appositely developed workbench with a spectrophotometer, is aware of the way the different coloured clothes to be recycled combine each other to create a new one. The tool has been validated using a set of 5,000 differently coloured clothes to be recycled and the classification error in classifying the clothes is within 5\%, i.e., lower than the one resulting from the use of an expert human operator.
\end{abstract}

